\title{
X Congreso Ibérico de Agroingeniería

\section{Predicción de la dinámica del carbono orgánico en suelos agrícolas del Cerrato Palentino (España) aplicando el modelo RothC}

\author{
Norlan M. Ruiz-Potosme1, Luis F. Sánchez-Sastre², Pablo Martín-Ramos, Carmen T. Bravo \\ Sánchez ${ }^{4}$, Mercedes Sánchez Báscones ${ }^{4}$, Salvador Hernández-Navarro², Jesús Martín-Gil² \\ 1 Departamento de Enseñanzas Técnicas, Universidad Europea Miguel de Cervantes, Valladolid, \\ España; nmruiz@uemc.es. \\ 2 Departamento de Ingeniería Agroforestal, ETSIIAA, Universidad de Valladolid, Palencia, España. \\ 3 Instituto Universitario de Investigación en Ciencias Ambientales de Aragón (IUCA), EPS, Universidad \\ de Zaragoza, Huesca, España \\ 4 Departamento de Ciencias Agroforestales, ETSIIAA, Universidad de Valladolid, Palencia, España
}

\begin{abstract}
Resumen: La gestión de los suelos agrícolas tiene un gran potencial para el secuestro de carbono, la reducción de las emisiones de gases de efecto invernadero y la consecución de una agricultura sostenible. Para predecir la acumulación de materia orgánica en suelos agrícolas del Cerrato Palentino (Palencia, España) se ha utilizado el modelo RothC, un método para simular a escala regional los cambios del carbono orgánico del suelo (COS) en sistemas agrícolas y forestales utilizando datos de clima y suelo. Con datos estimados de la materia orgánica inerte y la entrada mensual de vegetación o de residuos de la planta se han determinado los porcentajes de carbono inicial, carbono orgánico total anual y error frente al carbono orgánico experimental. El incremento de los contenidos de carbono orgánico en el periodo 2012-2013 ha oscilado entre el 4,9 y el $22.5 \%$, según parcela. Las predicciones de acumulación de carbono para 100 años han sido estimadas en torno al $70 \%$ para 4 de las cinco parcelas estudiadas y del $115 \%$ para la quinta. Esta información representa una herramienta útil para la planificación futura, en nuestra área geográfica, del uso de la tierra y su gestión en relación con la reducción de las emisiones de $\mathrm{CO}_{2}$
\end{abstract}

Palabras clave: entrada de C; materia orgánica inerte; modelo predictivo; RothC-26.3; secuestro de carbono

\section{Introducción}

Los balances de carbono a nivel mundial están cambiando significativamente, producto de la actividad antrópica [1]. Las cantidades de $\mathrm{CO}_{2}$ (gas de efecto invernadero) emitidas a la atmósfera, resultantes de la quema de combustibles fósiles y el cambio del uso de la tierra, están influyendo en el cambio de temperatura en el planeta Tierra y han generado una preocupación alrededor del fenómeno de cambio climático [1,2]. Una amenaza principal del cambio climático global es que afectaría a la producción de cultivos (por la alteración radical de los regímenes de temperaturas y lluvias) creando una situación comprometida para la seguridad alimentaria tanto a nivel local como mundial. 


\section{CONGRESO IBÉRICO DE AGROINGENIERÍA \\ X CONGRESSO IBÉRICO DE AGROENGENHARIA \\ 3 - 6 septiembre 2019, Huesca - España}

Este fenómeno cobra importancia en cuanto el proceso de secuestro de carbono por parte de los ecosistemas (sean estos "naturales" o agroecosistemas) resta en parte la indeseada acumulación atmosférica de $\mathrm{CO}_{2}$ generada por el excesivo uso de combustibles fósiles, principal causante del cambio climático [3].

Desde la agricultura, los estudios referentes a la captura de carbono surgen como un campo en la investigación acerca de la función que los sistemas agrícolas pueden cumplir frente al cambio climático [4]. En zonas agrícolas, reducir las emisiones de $\mathrm{CO}_{2}$ es sinónimo de aumentar la materia orgánica del suelo [5]. Hoy día, está ampliamente demostrado que la adopción de ciertas prácticas de manejo permite aumentar el carbono orgánico almacenado con el fin de aprovechar la gran capacidad que presentan los suelos agrícolas como sumidero [6-10]. Los sistemas de producción agrícola y en particular, los de conservación, presentan un gran potencial para capturar y almacenar carbono [11,12], generando efectos positivos adicionales: los suelos con mayor cantidad de materia orgánica tendrán mejor capacidad de infiltración del agua, mejor estructuración, resistirán mejor los procesos erosivos y brindarán beneficios a la productividad y sostenibilidad agrícola $[13,14]$. A su vez, en un contexto de cambio climático, el foco de los estudios recientes sobre la materia orgánica de los suelos apunta a determinar no solo su impacto sobre la productividad sino su función como posible destino del carbono de la atmósfera $[15,16]$.

Actualmente, el secuestro de carbono es uno de los servicios ecosistémicos de mayor interés en suelos cultivados y no cultivados. Muchos estudios han tratado de estimar la capacidad de secuestro de carbono de los suelos según las condiciones climáticas, edáficas y de manejo [17, 18], siendo el balance entre las entradas y salidas de carbono al sistema el determinante de la tasa de fijación de carbono en cada caso. De hecho, la adición de residuos vegetales, como los restos de cultivo, contribuyen a la emisión de otros gases de efecto invernadero que deben ser computados a la hora de realizar el balance global $[19,20]$. Si bien el almacenaje de carbono mide la diferencia acumulada en el suelo, el término carbon sequestration (para un periodo de tiempo concreto y un lugar determinado) debe ser considerado como el balance neto de todos los gases de efecto invernadero, expresado en equivalentes de $\mathrm{CO}_{2}$ o C- $-\mathrm{CO}_{2}$, teniendo en cuenta todas las fuentes de emisión en el sistema suelo-planta-atmósfera [21] (Figura 1). Toda vez que, en las tierras de cultivo, el tamaño de la acumulación de biomasa $C$ puede considerarse constante en una escala de décadas o siglos, el aumento de $C$ en el suelo a través de la adición de materia orgánica (MO) a las tierras de cultivo, puede conducir a una disminución del $\mathrm{CO}_{2}$ atmosférico. Por otra parte, es preciso recordar que el contenido de $\mathrm{C}$ en el suelo es uno de sus índices básicos de productividad [22].

En España, los bajos niveles de carbono orgánico registrados en suelos de clima mediterráneo y sobre todo, semiáridos, los sitúan a menudo por debajo de los valores propuestos como umbrales de degradación [23]. Según Romanyá et al. [24], del análisis comparativo del carbono entre suelos forestales y agrícolas de España se determinó que "los suelos de clima semiáridos presentan una mayor resistencia a la pérdida de carbono y una menor resiliencia que los suelos de climas más húmedos. Este hecho hace pensar que los suelos semiáridos pueden estar cerca de los umbrales de degradación y que por lo tanto su gestión debería evitar las pérdidas de materia orgánica". En el ciclo terrestre del carbono, el carbono orgánico del suelo representa la mayor reserva en interacción con la atmósfera y se estima en unos $1500 \mathrm{Pg}$ C a $1 \mathrm{~m}$ de profundidad y cerca de 2456 a $2 \mathrm{~m}$ de profundidad). El carbono inorgánico, que representa cerca de $1700 \mathrm{Pg}$, es capturado en formas más estables tales como $\mathrm{CaCO}_{3}$. La vegetación $(650 \mathrm{Pg})$ y la atmósfera (750 Pg) almacenan menos cantidades que los suelos [25]. El secuestro de carbono se ha convertido, así, en una estrategia clave para reducir la concentración de $\mathrm{CO}_{2}$ atmosférico y contribuir a la mitigación del cambio climático [22].

Las implicaciones sobre el cambio climático global del carbono almacenado en el suelo son, precisamente, las que han llevado a que su medición y estimaciones en diversos tipos de suelos (principalmente, agrícolas) haya cobrado importancia a nivel nacional y mundial [26-29]. 


\section{CONGRESO IBÉRICO DE AGROINGENIERÍA \\ X CONGRESSO IBÉRICO DE AGROENGENHARIA \\ 3 - 6 septiembre 2019, Huesca - España}

Entre los sistemas de predicción de almacenamiento de carbono orgánico del suelo los más utilizados a escalas regional y nacional han sido los modelos RothC [30-36], tanto para suelos forestales como agrícolas. Cabe destacar que el uso de estos modelos constituye un método alternativo y complementario a las técnicas experimentales a la hora de predecir resultados a medio y largo plazo, con el propósito de cuantificar el secuestro de $\mathrm{C}$, variación de materia orgánica y la reducción de las emisiones de $\mathrm{CO}_{2}$, de los suelos agrícolas.

Según Ruiz-Potosme [37] y Ruiz et al. [38], el modelo RothC-26.3 es capaz de predecir el porcentaje de carbono de un suelo (p.ej., conocer cuánto carbono orgánico del suelo a largo plazo puede estar disponible para un determinado uso, sea agrícola y forestal). Por ejemplo, a partir del modelo de RothC-26.3, podemos predecir a 100 años, el incremento de porcentaje de carbono orgánico del suelo. Publicaciones recientes reflejan la utilidad del modelo, entre los que cabe destacar el estudio de Romanya et al. [24] en suelos españoles.

El presente trabajo tiene como objetivo estimar y predecir el secuestro de carbono en suelos agrícolas en el Cerrato Palentino (España) aplicando el modelo preconizado RothC-26.3.

\section{Materiales y métodos}

Las parcelas objeto de estudio dentro del área geográfica seleccionada han sido ubicadas mediante el sistema de coordenadas Universal Transversal de Mercator (UTM), según distribución que figura en la Tabla 1. Su proyección cartográfica aparece en la Figura 1, donde son identificadas por las abreviaturas de los municipios a los que corresponden: $\mathrm{V}$ : Villamediana, SC: Soto de Cerrato, HC: Hérmedes de Cerrato, EC: Espinosa de Cerrato y VP: Valbuena de Pisuerga, todos pertenecientes al Cerrato Palentino. Esta área geográfica se localiza en el sudeste de la provincia de Palencia y se caracteriza por una estructura geológica formada por depósitos del Mioceno (principalmente de margas, yesos y calizas), suelos pertenecientes a los tipos entisoles, inceptisoles-Xerochrepts y alfisoles, altos rendimientos en secano y, especialmente, en regadío (principalmente, remolacha y patata) y un clima semiárido y subárido, con escasez de lluvia y temperaturas altas en verano y frías en invierno.

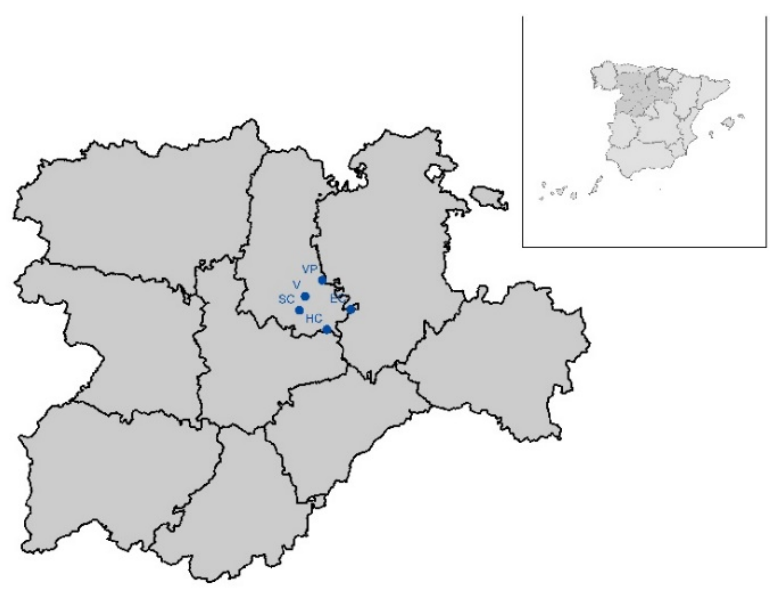

Figura 1. Proyección cartográfica de las parcelas experimentales, cuyas siglas VP: Valbuena de Pisuerga, V: Villamediana, SC: Soto de Cerrato, EC: Espinosa de Cerrato y HC: Hérmedes de Pisuerga. Fuente: Instituto Geográfico Nacional (IGN-1986). 


\section{CONGRESO IBÉRICO DE AGROINGENIERÍA \\ X CONGRESSO IBÉRICO DE AGROENGENHARIA \\ 3- 6 septiembre 2019, Huesca - España}

Tabla 1. Ubicación geográfica (coordenadas UTM), de las cinco parcelas experimentales objeto de estudio. Fuente: Instituto Geográfico Nacional (IGN-1986).

\begin{tabular}{ccccccc}
\hline Ubicación de las parcelas & Coordenadas X Coordenadas $Y$ & Longitud & Latitud & Altitud \\
\hline Villamediana & 386200 & 4655000 & $4^{\circ} 22^{\prime} 30^{\prime \prime} \mathrm{W}$ & $42^{\circ} 2^{\prime} 20^{\prime \prime} \mathrm{N}$ & $820 \mathrm{~m}$ \\
Soto de Cerrato & 381900 & 4645000 & $4^{\circ} 25^{\prime} 29^{\prime \prime} \mathrm{W}$ & $41^{\circ} 56^{\prime} 53^{\prime \prime} \mathrm{N}$ & $725 \mathrm{~m}$ \\
Hérmedes de Cerrato & 402700 & 4630000 & $4^{\circ} 10^{\prime} 17^{\prime \prime} \mathrm{W}$ & $41^{\circ} 48^{\prime} 57^{\prime \prime} \mathrm{N}$ & $905 \mathrm{~m}$ \\
Espinosa de Cerrato & 420800 & 4646000 & $3^{\circ} 57^{\prime} 21^{\prime \prime} \mathrm{W}$ & $41^{\circ} 57^{\prime} 43^{\prime \prime} \mathrm{N}$ & $890 \mathrm{~m}$ \\
Valbuena de Pisuerga & 399100 & 466800 & $4^{\circ} 13^{\prime} 17^{\prime \prime} \mathrm{W}$ & $42^{\circ} 9^{\prime} 27^{\prime \prime} \mathrm{N}$ & $895 \mathrm{~m}$ \\
\hline
\end{tabular}

Las 5 parcelas objeto de estudio se utilizan como suelos agrícolas para cultivos de secano.

Las muestras de suelo fueron tomadas en las parcelas experimentales en el mes de julio de 2016, a una profundidad de $35 \mathrm{~cm}$, según Fallon et al [30,31], prescindiendo del horizonte $0 \mathrm{o}$ mantillo) en cantidades de aprox. $1 \mathrm{~kg}$, y subsiguientemente, analizadas. Las muestras fueron tamizadas para separar los gruesos (tamiz de $2 \mathrm{~mm}$ ) de los finos (tamiz de $0.1 \mathrm{~mm}$ ).

Con las muestras de suelos recogidas de las diferentes parcelas y mediante los métodos convencionales de la American Society for Testing Materials, se han determinado los valores de los parámetros edáficos: $\mathrm{pH}$, carbono orgánico, arcilla, limo y arena (Tabla 2). La densidad aparente para estos suelos es, en promedio, de $1,35 \mathrm{~g} \cdot \mathrm{cm}^{-3}$

Tabla 2. Parámetros edáficos experimentales para los 5 puntos de muestreo (CO en $\left.t \mathrm{ha}^{-1}\right)$

\begin{tabular}{ccccccc}
\hline No parcela & Municipio & pH & CO & \% Arcilla & \% Limo & \% Arena \\
\hline Parcela 1 & Villamediana & 8,85 & 4,80 & 8,10 & 6,25 & 88,60 \\
Parcela 2 & Soto de Cerrato & 8,95 & 8,25 & 8,65 & 56,15 & 38,25 \\
Parcela 3 & Hérmedes de Cerrato & 8,40 & 11,20 & 27,25 & 28,10 & 45,85 \\
Parcela 4 & Espinosa de Cerrato & 8,35 & 16,10 & 16,30 & 17,70 & 68,75 \\
Parcela 5 & Valbuena de Pisuerga & 8,65 & 22,50 & 19,50 & 28,80 & 54,55 \\
\hline
\end{tabular}

Los datos climáticos para la zona estudiada se encuentran recogidos en la Tabla 3. La temperatura del aire y la pluviometría como medias mensuales utilizadas han sido obtenidas de bases de datos procedentes de diferentes centros meteorológicos y corresponden a valores promedio de los últimos 30 años.

Tabla 3. Datos mensuales meteorológicos de la zona estudiada (provincia de Palencia)

\begin{tabular}{cccc}
\hline Mes & Temperatura $\left({ }^{\circ} \mathbf{C}\right)$ & Precipitación $(\mathbf{m m})$ & ETP $(\mathbf{m m})$ \\
\hline Enero & 2 & 64 & 6,60 \\
Febrero & 3 & 45 & 11,34 \\
Marzo & 5 & 75 & 26,15 \\
Abril & 8 & 57 & 41,10 \\
Mayo & 15 & 68 & 69,35 \\
Junio & 18 & 40 & 105,50 \\
Julio & 19 & 15 & 125,15 \\
Agosto & 24 & 12 & 106,20 \\
Septiembre & 13 & 36 & 76,61 \\
Octubre & 10 & 84 & 50,85 \\
Noviembre & 4 & 110 & 21,40 \\
Diciembre & 1 & 90 & 9,65 \\
\hline
\end{tabular}




\section{CONGRESO IBÉRICO DE AGROINGENIERÍA \\ X CONGRESSO IBÉRICO DE AGROENGENHARIA \\ 3 - 6 septiembre 2019, Huesca - España}

Para determinar el porcentaje de materia orgánica fácilmente oxidable se ha utilizado el Método de Walkley y Black [59] previa eliminación de los carbonatos por ignición a $360{ }^{\circ} \mathrm{C}$ (durante $2 \mathrm{~h}$ ), seguida de enfriamiento a $150{ }^{\circ} \mathrm{C}$ en la mufla. Este método, capaz de discriminar entre el humus y las formas fuertemente condensadas (entre las que se cuentan el grafito y el carbón vegetal), permite cuantificar la materia orgánica fácilmente oxidable a partir de la fracción de materia orgánica del suelo que reacciona con $\mathrm{K}_{2} \mathrm{Cr}_{2} \mathrm{O}_{7} 1 \mathrm{~N}$ en medio $\mathrm{H}_{2} \mathrm{SO}_{4}$ y durante 30 min. Se sabe que la acción del $\mathrm{H}_{2} \mathrm{SO}_{4}$ concentrado es capaz de producir la oxidación del carbono de la materia orgánica a $\mathrm{CO}_{2}$. El anión dicromato (añadido en exceso) y no reducido se analiza por titulación.

En la Figura 2, se ofrece la estructura del modelo de RothC que utiliza el cociente entre el material de planta descomponible (DPM) y el material de planta resistente (RPM), DPM/RPM, para determinar el grado de descomposición del material de planta entrante en el sistema a partir de la entrada de materia orgánica. El carbono orgánico del suelo (en t C ha-1) se divide en cuatro fracciones activas y una fracción pequeña de materia orgánica inerte (IOM). Las fracciones activas son: material de la planta descomponible (DPM), material de la planta resistente (RPM), biomasa microbiana (BIO) y materia orgánica húmica (HUM). Cada fracción descompone siguiendo una cinética de primer orden y con su velocidad característica. La fracción de IOM se considera resistente a la descomposición.

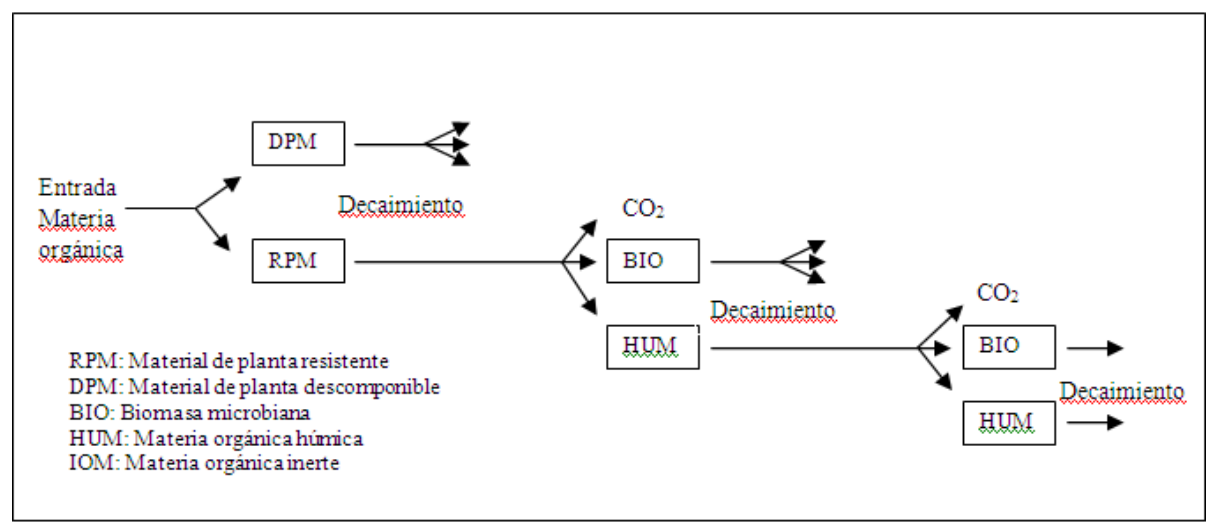

Figura 2. Estructura del modelo RothC. Adaptado de Coleman et al. [39,40]

Para la aplicación del método se contó con los registros climatológicos de la zona de estudio, ya que estas variables (temperatura, humedad, y precipitación) están íntimamente ligadas al proceso de descomposición de la materia orgánica.

Las estimaciones de IOM para las parcelas en estudio se obtuvieron por la ecuación de Fallon et al, [30]: IOM $=0.049 \times \mathrm{CO}^{1.139}$

Para calcular la entrada de C input, se utiliza el cociente DPM/RPM del material entrante de cada planta particular, cuyos valores asignados a cada resto vegetal fueron de 1.44 para suelos con cubierta vegetal. Todo el material que entra en la planta, origina tanto DPM como RPM, que a su vez se descomponen para formar $\mathrm{CO}_{2}$, biomasa y materia húmica (BIO+HUM). La proporción que da lugar al $\mathrm{CO}_{2}$ y la que origina la $\mathrm{BIO}+\mathrm{HUM}$, se estima a partir del contenido de arcilla del suelo, mediante el modelo RothC.

La predicción de la evolución del CO a corto y largo plazo se ha llevado a cabo utilizando el modelo RothC-26.3 [39-40], descrito en FORTRAN77, que es una extensión de un modelo previo descrito por Jenkinson y Rayner [39] y por Coleman et al., [40], que permite conocer cuánto carbono orgánico puede estar disponible para determinados usos. 


\section{CONGRESO IBÉRICO DE AGROINGENIERÍA \\ X CONGRESSO IBÉRICO DE AGROENGENHARIA \\ 3 - 6 septiembre 2019, Huesca - España}

\section{Resultados y discusión}

Como se ha dicho, el modelo Roth es capaz de predecir, a 100 años, el porcentaje de carbono de un suelo e incluso conocer cuánto carbono orgánico del suelo puede estar disponible para una determinada aplicación [37]. En la Tabla 4 se muestran los porcentajes de materia orgánica y toneladas de carbono oxidable junto a las toneladas de carbono orgánico total determinados por la metodología arriba indicada (método de Walkley y Black [59]), para las parcelas objeto de estudio.

Tabla 4. Porcentajes de materia orgánica oxidable (MO oxidable), toneladas de carbono oxidable por hectárea $\left(\mathrm{t} \mathrm{C} \mathrm{ha}^{-1}\right)$ y toneladas de carbono total por hectárea $(\mathrm{t} \mathrm{C} \mathrm{ha-1)} \mathrm{en} \mathrm{los} \mathrm{cinco} \mathrm{puntos} \mathrm{de}$ muestreo

\begin{tabular}{cccc}
\hline Punto de muestreo & \% MO oxidable (a) & $\mathbf{t ~ C ~}$ ha $^{-1}$ oxidable (b) & $\mathbf{t ~ C ~}$ ha $^{-1}$ total (c) \\
\hline Parcela 1 & 0,30 & 3,2 & 4,12 \\
Parcela 2 & 2,48 & 17,2 & 23,50 \\
Parcela 3 & 1,20 & 12,5 & 16,15 \\
Parcela 4 & 0,95 & 9,15 & 11,72 \\
Parcela 5 & 0,85 & 6,8 & 7,95 \\
\hline
\end{tabular}

Con los valores estimados para la materia orgánica inerte (IOM) y la entrada mensual de vegetación o de residuos de la planta (C input), recogidos en la tabla 5, es posible determinar la velocidad de secuestro de las parcelas objeto de estudio. Esta decrece a lo largo del tiempo y a medida que el sistema se aproxima a la situación de equilibrio o de saturación del suelo.

Tabla 5. Valores estimados de la materia orgánica inerte (IOM) y de la entrada de carbono (C input) en las parcelas objeto de estudio.

\begin{tabular}{cccc}
\hline Punto de muestreo & IOM & C Input & Edad de las áreas de suelos agrícolas \\
\hline Parcela 1 & 0,5 & 0,050 & 5 \\
Parcela 2 & 0,5 & 0,155 & 20 \\
Parcela 3 & 0,5 & 0,120 & 35 \\
Parcela 4 & 0,5 & 0,095 & 15 \\
Parcela 5 & 0,5 & 0,085 & 50 \\
\hline
\end{tabular}

Los porcentajes de carbono inicial y carbono orgánico total en el año 2013 junto con la estimación del error frente al carbono orgánico experimental se encuentran recogidos en la Tabla 6.

Tabla 6. Porcentajes experimental y calculado de carbono orgánico total.

\begin{tabular}{ccccc}
\hline Punto de muestreo & $\begin{array}{c}\text { \% C inicial / } \\
\text { año }\end{array}$ & $\begin{array}{c}\text { \% C total exp. } \\
\mathbf{2 0 1 3}\end{array}$ & $\begin{array}{c}\text { \% C total calculado } \\
\mathbf{2 0 1 3}\end{array}$ & \% Error C \\
\hline Parcela 1 & $3,85(2008)$ & 4,86 & 4,90 & 0,04 \\
Parcela 2 & $5,15(1993)$ & 22,54 & 22,60 & 0,06 \\
Parcela 3 & $4,65(1978)$ & 15,94 & 15,90 & 0,04 \\
Parcela 4 & $4,85(1998)$ & 17,33 & 17,08 & 0,25 \\
Parcela 5 & $4,45(1963)$ & 8,96 & 8.75 & 0,21 \\
\hline
\end{tabular}

Se observa que la velocidad inicial del incremento del secuestro de carbono por año es aproximadamente 22.6 en la parcela 2, correspondiente al municipio de Soto de Cerrato. 


\section{CONGRESO IBÉRICO DE AGROINGENIERÍA \\ X CONGRESSO IBÉRICO DE AGROENGENHARIA \\ 3 - 6 septiembre 2019, Huesca - España}

Es preciso destacar que, a largo plazo, la productividad puede verse incrementada en zonas de secano y la precipitación limita la producción, pero también disminuye la descomposición, resultando en mayores incrementos en el carbono orgánico del suelo, por lo que también aumentan las entradas de carbono al suelo [42,43].

En la tabla 7 se muestran las predicciones a 100 años.

Tabla 7. Valores de carbono orgánico teóricos y predicciones a 100 años.

\begin{tabular}{ccc}
\hline Carbono teórico, 2008 (t C/ha) & Predicción a 100 años (t C/ha) & \% Incremento (100 años) \\
\hline 4,90 & 9,45 & 115,70 \\
22,60 & 35,55 & 52.58 \\
15,90 & 25,85 & 60,52 \\
17,08 & 19,80 & 84,31 \\
8.75 & 14,12 & 78,50 \\
\hline
\end{tabular}

En las Figuras 3 y 4 se observa el especial incremento de carbono orgánico total previsto para al año 2013 obtenido para la parcela 2, ubicada en Soto de Cerrato, Palencia, con una edad de suelos agrícolas de 20 años. Es seguido por el obtenido para la parcela 3 (Hérmedes de Cerrato), con edad de suelos agrícolas de 35 años, luego por el de la parcela 4 (Espinosa de Cerrato), con edad de implantación de cultivos agrícolas de 15 años y finalmente, por el de la parcela 5 (Valbuena de Pisuerga), con edad de 50 años, durante 1965-2015.

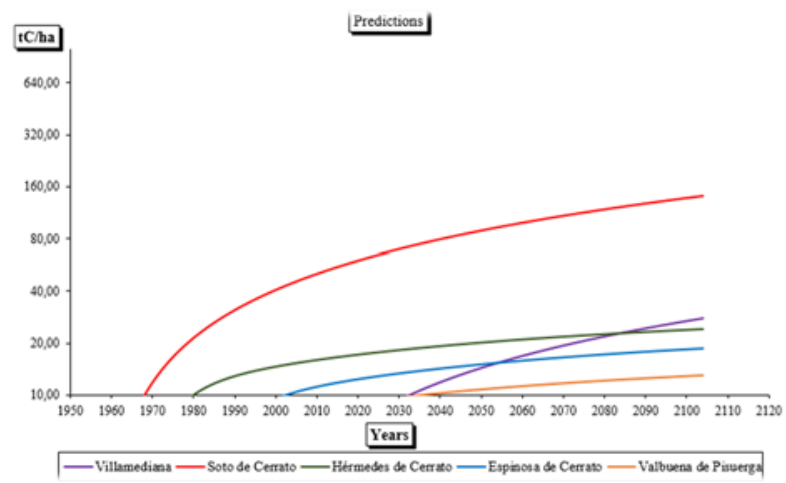

Figura 1. Datos estimados de predicciones de carbono a 100 años de cada parcela agrícola de estudio.

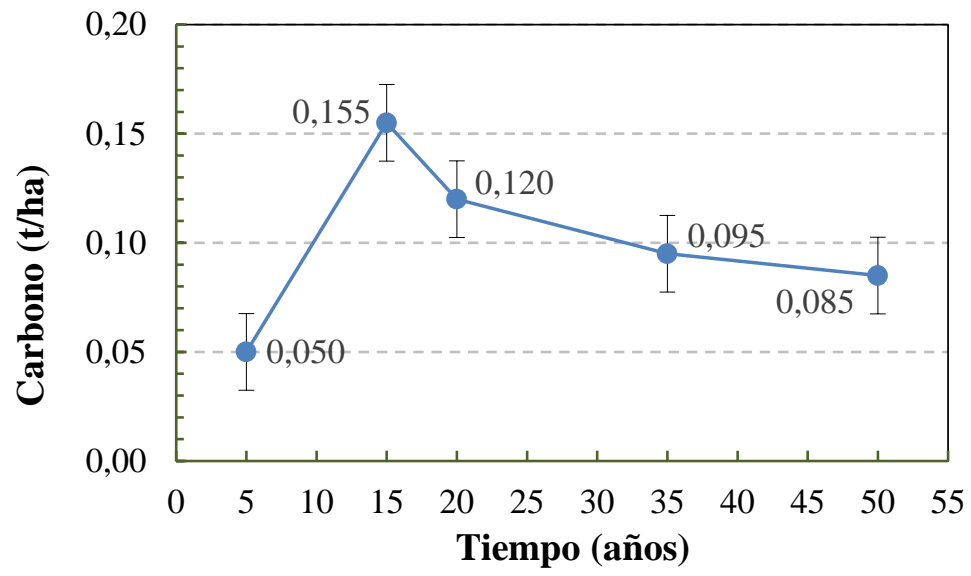

Figura 2. Estimación de la entrada de carbono ( $\mathrm{C}$ input en $\left.\mathrm{t} \mathrm{C}^{\mathrm{h}} \mathrm{a}^{-1}\right)$ en función de los años, para las parcelas estudiadas 


\section{CONGRESO IBÉRICO DE AGROINGENIERÍA \\ X CONGRESSO IBÉRICO DE AGROENGENHARIA \\ 3 - 6 septiembre 2019, Huesca - España}

Es preciso tener en cuenta que las emisiones de gases de efecto invernadero de la agricultura, la silvicultura y pesca casi han duplicado en los últimos 50 años y que las transformaciones del uso del suelo y el drenaje de suelos orgánicos para el cultivo son responsables de aproximadamente el $11 \%$ de todas las emisiones de gases de efecto invernadero (GEI). También se estima que los GEI aumentarán en las próximas décadas debido a la demanda creciente de alimentos. No obstante, la mejora de prácticas del cultivo y las nuevas tecnologías emergentes permitirán una reducción de emisiones por unidad de alimento producida [44].

Al restaurar los suelos degradados y adoptar prácticas de manejo sostenible como la rotación de cultivos, la labranza cero, la agricultura de conservación, la agrosilvicultura y la agroecología, existe el potencial para disminuir los GEI procedentes de la agricultura, mejorar la retención de carbono y aumentar la resiliencia ante el cambio climático [33]. Si se gestionan de forma sostenible, los suelos pueden ser parte de la solución cuando se trata de la mitigación del cambio climático [45-49].

Actualmente, parece existir consenso en que la adopción de ciertas prácticas de manejo (RMPs - recommended management practices) permite aumentar el carbono orgánico almacenado aprovechando la gran capacidad que presentan los suelos agrícolas como sumidero [9, 50-55].

\section{Conclusiones}

El estudio realizado ha conseguido mostrar la viabilidad del modelo de RothC para estimar y simular los efectos del manejo agrícola en la dinámica del secuestro de carbono orgánico (SOC) en las parcelas objeto de estudio. Se ha evidenciado que tanto el incremento del cultivo como la reducción de la intensidad de la labranza son estrategias efectivas para aumentar el secuestro de SOC en condiciones semiáridas, que podrían compensar las emisiones antropogénicas de $\mathrm{CO}_{2}$.

No obstante, el método considerado también tiene ciertas restricciones y debilidades, por ejemplo, las relacionadas con la resolución de datos estadísticos sobre agricultura, que deben estar vinculados a datos de puntos de suelo o polígonos. Esta es una fuente importante de incertidumbre en términos de valores absolutos de stock de $\mathrm{C}$ modelado por lo que su aplicación resulta delimitada para la predicción del cambio de $C$ en un lugar determinado.

En nuestra investigación, tanto el modelo RothC como el análisis comparativo del suelo en los puntos georreferenciados son concluyentes sobre el aumento del stock de $\mathrm{C}$ durante el período 1965-2015.

Sin embargo, los experimentos a largo plazo, que son indispensables, no pueden cubrir la gran diversidad de opciones de manejo disponibles para los agricultores. Se necesita un enfoque participativo que enfrente la dificultad de medir de manera confiable los cambios en las existencias de SOC en una escala de campo de solo unos pocos años, que resulte relevante para el agricultor [56].

Estimamos que la aplicación de medidas realistas para mejorar la retención de residuos de cultivos, el uso de cultivos de cobertura y la conversión a pastizales mejoraría el secuestro de carbono del suelo correspondiente a la compensación de emisiones en 0.50 millones de toneladas de $\mathrm{CO}_{2}$ por año, que solo corresponde al $5 \%$ de las emisiones agrícolas actuales de metano y óxido nitroso [57].

En lo que respecta a España, los datos disponibles indican que en los primeros $30 \mathrm{~cm}$ los suelos agrícolas (cultivos anuales y cultivos leñosos) acumulan el 31,9\% del COS total nacional [58], porcentaje por encima de la media mundial debido en gran medida a que el 33,5\% de la superficie española está ocupada por cultivos [58]. 


\section{CONGRESO IBÉRICO DE AGROINGENIERÍA \\ $X$ CONGRESSO IBÉRICO DE AGROENGENHARIA \\ 3 - 6 septiembre 2019, Huesca - España}

\section{Referencias}

1. Huang, Y.; Yu, Y.; Zhang, W.; Sun, W.; Liu, S.; Jiang, J.; Wu, J.; Yu, W.; Wang, Y. \& Yang, Z. Agro-C: A biogeophysical model for simulating the carbon budget of agroecosystems. Agricultural and Forest Meteorology. Agricultural and Forest Meteorology, 2009, 149, $106-129$

2. Easterling, WE.; Aggarwal, PK.; Batima, P.; Brander, KM.; Erda, L.; Howden, SM.; Kirilenko, A.; Morto, J.; Soussana, JF.; Schmidhuber, J.; Tubiello, FN. Food, fibre and forest products. Climate Change 2007: Impacts, Adaptation and Vulnerability: Contribution of Working Group II to the Fourth Assessment Report of the Intergovernmental Panel on Climate Change (Parry ML et al, eds). Cambridge, UK. 2007, Cambridge University Press, 273-313 pp.

3. Stavins, R.; \& Richards, K. The cost of U.S. forest based: Carbon Sequestration. Economics. Pew Center on Global Climate Change. 2005, 40 p.

4. West, T. Net Sequestration Carbon in Agriculture: A national Assessment. Environmental Sciences Division, Oak Ridge National Laboratory. 2008, pp. 1 -6.

5. Jarecki, MK.; Lal, R. Crop management for soil carbon sequestration. Critical Reviews in Plant Sciences, 2003, 22(5), 471-502.

6. Paustian, K.; Andrén, O.; Janzen, HH.; Lal, R.; Smith, P.; Tian, G.; Tiessen, H.; Van Noordwijk, M.; Woomer, PL. Agricultural soils as a sink to mitigate CO2 emissions. Soil Use and Management, 1997, 13, 230-244.

7. Smith, P.; Powlson, DS.; Smith, JU.; Falloon, P.; Coleman, K. Meeting Europe's climate change commitments: quantitative estimates of the potential for carbon mitigation by agriculture. Global Change Biology, 2000, 6, 525-539.

8. Kimble, JM.; Lal, R.; Follett, RF. Agricultural practices and policies for carbon sequestration in soil. Lewis Publishers, Boca Raton, FL, 2002.

9. Freibauer, A.; Rounsevel, MDA.; Smith, P.; Verhagen, J. Carbon sequestration in the agricultural soils of Europe. Geoderma, 2004, 122, 1-23.

10. Nieto, O.M.; Castro, J.; Fernández, E. Simulation of soil organic carbon stocks in a Mediterranean olive grove under different soil-management systems using the RothC model. Soil Use and Management, 2010, 26, 118-125.

11. LaSalle, J. T. Hepperly, P. Regenerative Organic Farming: A solution to Global Warming Rodale Institute. 2008, pp. 1-9.

12. Dendooven, L.; Patiño-Zuñig, L.; Luna-Guido, M.; Marsch, R.; Govaerts, B. Capítulo 1 Ecosistemas Terrestres. 1.63. Global Warming Potential of agricultural systems with contrasting tillage and residue management in the central highlands of Mexico. In: Memorias del III Simposio Internacional del Carbono en México. Universidad Autónoma del Estado de México-Programa Mexicano del Carbono-Instituto Nacional de Ecología, México. 2011, pp. 778.

13. Altieri, M. Agroecology: the science of Natural Resource Management for poor farmers in marginal environments. Agriculture, Ecosystems and Environment, 2002, 93, 1-24.

14. Robbins, M. Crops and Carbon: Paying Farmers to Combat Climate Change. Earth scan, Great Britain. 2001, 300 pp.

15. Mishra, U.; Lal, R.; Slater, B.; Calhoun, F.; Liu, D.; Van Meirvenne, M. Predicting soil organic carbon stock using profile depth distribution functions and ordinary kriging. Soil Sci. Soc. Am. J., 2009, 73, 614-621.

16. Meersmans, J.; Van Wesemael, B.; De Ridder, F.; Van Molle, M. Modelling the three-dimensional spatial distribution of soil organic carbon (SOC) at regional scale (Flanders, Belgium). Geoderma, 2009, 152, 43-52.

17. Schulp, C.J.E.; Nabuurs, G.J.; Verburg, P.H. Future carbon sequestration in Europe - Effects of land use change. Agric. Ecosys. Environ., 2008, 127, 251-264.

18. Liang, B.C.; Campbell, C.A.; McConkey, B.G.; Padbury, G.; Collas, P. An empirical model for estimating carbon sequestration on the Canadian prairies. Can. J. Soil Sci., 2005, 85, 549-556.

19. Porta, J.; López-Acevedo, M.; Roquero, C. (eds.). Edafología para la agricultura y el medio ambiente. $3^{\underline{a}}$ ed. Mundi-Prensa, Madrid, 2003, 929 p.

20. Dawson, J.J.C.; \& Smith, P. Carbon losses from soil and its consequences for land-use management. Science of the Total Environment 2007, 382, 165-190. 


\section{CONGRESO IBÉRICO DE AGROINGENIERÍA \\ $X$ CONGRESSO IBÉRICO DE AGROENGENHARIA \\ 3- 6 septiembre 2019, Huesca - España}

21. Feller, C.; Bernoux, M. Historical advances in the study of global terrestrial soil organic carbon sequestration. Waste Management, 2008, 28(4), 734-740.

22. Lal, R. Soil carbon sequestration impacts on global climate change and food security, Science, 2004, 304, 1623-1627.

23. Romanyà, J.; Rovira, P.; Duguy, B.; Vallejo, R.; Rubio, A. C sequestration issues in the Mediterranean soils, in: Greenhouse-gas Budget of Soils under Changing Climate and Land Use (Burnout), edited by: Jandl, R. and Olsson, M., BFW, 2007, 15-22.

24. Romanyà, J.; Cortina, J.; Falloon, P.; Coleman, K.; Smith, P. Modelling changes in soil organic matter after planting fast-growing Pinus radiata on Mediterranean agricultural soils. European Journal of Soil Science 2000, 51, 627-641.

25. Charro E.; Hernández-Navarro S.; Martín-Gil J.; Moyano A.; Ruiz-Potosme N. Estimación del secuestro de carbono en suelos bajo masas forestales de Pinus halepensis en Castilla y León (España). 2ª Reunión del Grupo de Trabajo de Ecología, Ecofisiología y Suelos Forestales, Sociedad Española de Ciencias Forestales (SECF), Salamanca (Spain), 2007, 284 pp.

26. Segura-Castruita, M.A; Sánchez-Guzmán, P; Ortiz -Solorio, C. A.; Gutiérrez -Castorena, Ma. del C. Carbono orgánico de los suelos de México. Terra Latinoamericana, 2005, 23: 21-28.

27. Álvarez, G.; Krasilnikov, P.; García-Calderón, N.E. Vertical distribution and soil organic matter composition in a montane cloud forest, Oaxaca, Mexico. European Journal of Forest Research, 2012, 6, 16431651.

28. Gamboa, A.M.; Hidalgo, C.; F. De Leon, J. D.; Etchevers, J. F.; Gallardo Campo. J. Nutrient Addition Differentially Affects Soil Carbon Sequestration in Secondary Tropical Dry Forests: Early- versus LateSuccession Stages. Restoration Ecology, 2008, 18, 252-260.

29. Chiti, T.; Gardin, L.; Perugini, L.; Quaratino, R.; Primo-Vaccari, F.; Migliett, F.; Valentini, R. Soil organic carbon stock assessment for the different cropland land uses in Italy. Biology and Fertility of Soils, 2012, 48, 9-17.

30. Falloon, P.; Smith, P.; Coleman, K.; Marshall, S. Estimating the size of the inert organic matter pool from total soil organic carbon content for use in the Rothamsted carbon model. Soil Biology and Biochemistry, 1998, 30, 1207-1211.

31. Falloon P; Smith P. Simulating SOC changes in long-term experiments with RothC and CENTURY: model evaluation for a regional scale application. Soil Use and Management, 2002, 18, 101-111.

32. Falloon, P.; Smith, P. Accounting for changes in soil carbon under the Kyoto Protocol: need for improved long-term data sets to reduce uncertainty in model projections. Soil Use and Management, 2003, 19, 265269.

33. Smith, J.; Smith, P.; Wattenbach, M.; Zaehle, S.; Hiederer, R.; Jones, R.J.A.; Montanarella, L.; Rounsevell, M.; Reginster, I.; Ewert, F. Projected changes in mineral soil carbon of European croplands and grasslands, 1990-2080. Global Change Biology, 2005, 11, 2141-2152.

34. Smith, J.; Smith, P.; Wattenbach, M.; Gottschalk, P.; Romanenkov, V.A.; Ševcova, L.K.; Sirotenko, O.D.; Rukhovič, D.I.; Koroleva, P.V.; Romanenko, I.A.; Lisovoj, N.V. Projected changes in the organic carbon stocks of cropland mineral soils of European Russia and the Ukraine 1990-2070. Global Change Biology, 2007, 13, 342-354.

35. Van Wesemael, B.; Lettens, S.; Roelandt, C.; Van Orshoven, J. Modelling the evolution of regional carbon stocks in Belgian cropland soils. Canadian Journal of Soil Science, 2005, 85, 511-521.

36. Easter, M.; Paustian, K.; Killian, K.; Williams, S.; Freng, T.; Al-Adamat, R.; Batjes, N.H.; Bernoux, M.; Bhattacharyya, T.; Cerri, C.C.; Cerri, C.E.P.; Coleman, K.; Falloon, P.; Feller, C.; Gicheru, P.; Kamoni, P.; Milne, E.; Pal, D.K.; Powlson, D.S.; Rawajhif, Z. The GE FSOC soil carbon modeling system: A tool for conducting regional-scale soil carbon inventories and assessing the impacts of land change on soil carbon. Agriculture, Ecosystems \& Environment, 2007, 122, 13-25.

37. Ruiz Potosme, N.M. Determinación de la distribución potencial de Pinus Halepensis Mill en Castilla y León, mediante técnicas geoestadísticas. Tesis Doctoral, Escuela Técnica Superior de Ingenierías Agrarias, Campus de Palencia - Universidad de Valladolid, España, 2009.

38. Ruiz, N.M.; Bravo, T.B.; Sánchez, L.F.; Correa Guimaraes, A.; Martín Gil, J. Application of RothC Model, in forest soil of Castilla y Leon, Spain. II Congreso do solo, Viçosa-MG, Brasil, 2013, 10-12 abril. 


\section{CONGRESO IBÉRICO DE AGROINGENIERÍA \\ X CONGRESSO IBÉRICO DE AGROENGENHARIA \\ 3 - 6 septiembre 2019, Huesca - España}

39. Jenkinson, D.S.; Rayner, J.H. The turnover of soil organic matter in some of the Rothamsted classical experiments. Soil Sci. 1977, 123, 298-305.

40. Coleman, K.; \& Jenkinson, D.S. RothC-26.3. A model for the turnover of carbon in soil. In D.S. Powlson, P.; Smith J.U.; Smith eds. Evaluation of soil organic matter models using existing long-term datasets. NATO ASI Series I, Springer-Verlag, Heidelberg, 1995, 38, 237-246.

41. Smith, J.U.; Smith, P.; Wattenbach, M.; Zaehle, S.; Hiederer, R.; Jones, R.J.A.; Montanarella, L.; Rounsevell, M.; Reginster, I.; Ewert, F. Projected changes in mineral soil carbón of European croplands and grasslands, 1990-2080. Global Change Biology, 2005, 11, 2141-2152.

42. Jobbágy, E.G.; \& Jackson, R.B. The vertical distribution of soil organic carbon and its relation to climate and vegetation. Ecological Applications, 2000, 10 (2), 423- 436.

43. López-Bellido, L. Agricultura, Cambio Climático y Secuestro de Carbono, Editor: Createspace Edición 1, 2015, ISBN-13: 9781507735398.

44. Organización de las Naciones Unidas para la Alimentación y la Agricultura, FAO. Mitigación del cambio climático y adaptación en la agricultura, la silvicultura y la pesca. Departamento de Gestión de Recursos Naturales y Medio Ambiente, 2015, Roma, Italia.

45. Wu H.; Guo Z.; Peng C. Land use induced changes of organic carbon storage in soils of China. Glob Change Biol. 2003, 9, 305-15.

46. Maia, S.M.F.; Ogle S.M.; Cerri C.E.P.; Cerri C.C. Soil organic carbon stock change due to land use activity along the agricultural frontier of the southwestern Amazon, Brazil, between 1970 and 2002. Glob Change Biol. 2010, 16, 2775-88.

47. Sanz-Cobena, A.; Lassaletta L.; Garnier, J.; Smith, P. Mitigation and quantification of greenhouse gas emissions in Mediterranean cropping systems. Agriculture, Ecosystems E Environment, 2017, 238, 1-4.

48. MMAMRM (2008): Plan Nacional de Adaptación al Cambio Climático (PNACC). Centro de Publicaciones de la Secretaría General Técnica del Ministerio de Medio Ambiente y Medio Rural y Marino, Madrid.

49. Lal, R. Residue management, conservation tillage and soil restoration for mitigating greenhouse effect by $\mathrm{CO}_{2}$ enrichment. Soil and Tillage Research, 1997, 43, 81-107.

50. Paustian, K.; Six, J.; Elliott, E.T.; Hunt, H.W. Management options for reducing $\mathrm{CO}_{2}$ emissions from agricultural soils. Biogeochemistry, 2000, 48, 147-163.

51. Hutchinson, J.J.; Campbell, C.A.; Desjardins, R.L. Some perspectives on carbon sequestration in agriculture. Agricultural and Forest Meteorology, 2007, 142, 288-302.

52. Smith, P.; Martino, D.; Cai, Z.; Gwary, D.; Janzen, H.; Kumar, P.; McCarl, B.; Ogle, S.; O'Mara, F.; Rice, C.; Scholes, B.; Sirotenko, O.; Howden, M.; McAllister, T.; Pan, G.; Romanenkov, V.; Schneider, U.; Towprayoon, S. Policy and technological constraints to implementation of greenhouse gas mitigation options in agriculture. Agriculture, Ecosystems and Environment, 2007, 118, 6-28.

53. Farina, R.; Marchetti, A.; Francaviglia, R.; Napoli, R.; Di Bene, C. Modeling regional soil C stocks and $\mathrm{CO}_{2}$ emissions under Mediterranean cropping systems and soil types. Agriculture, Ecosystems and Environment, 2017, 238, 128-141.

54. Kaczynski, R., Siebielec, G., Marjoleine C. H., Korevaar, H., (2017): Modelling soil carbon trends for agriculture development scenarios at regional level. Geoderma, 2017, 286, 104-115.

55. Perez, C.; Roncoli, C.; Neely, C; Steiner J.L. Can carbon sequestration markets benefit low-income producers in semi-arid Africa? Potentials and challenges. Agric. Syst. 2007, 94, 2-12.

56. Taghizadeh-Toosi, A.; Olesen, J.E. Modelling soil organic carbon in Danish agricultural soils suggests low-potential for future carbon sequestration. Agric. Syst., 2016, 145, 83-89.

57. Rodriguez Martin, L.A; Álvaro-Fuentes, J; Gonzalo, J; Gil, C; Ramos-Miras, J.J; Grau Corbí, J.M; Bolud, R. Assessment of the soil organic carbon stock in Spain. Geoderma, 2016, 264, 117-125.

58. ESYRCE, (2015): Estadística sobre superficies y rendimientos de cultivos. http://www. magrama.gob.es/es/estadistica/temas/estadisticas-agrarias/agricultura/esyrce/ Accessed: 10 March 2016.

59. Walkley, S.J.; Black, I.A. An examination of the Degtjareff method for determining soil organic matter and a proposed modification of the chromic acid titration method. Soil Sci., 1934, 37, 29-38. 